\title{
Oseltamivir reduces hippocampal abnormal EEG activities after a virus infection (influenza) in isoflurane-anesthetized rats
}

This article was published in the following Dove Press journal:

Journal of Experimental Pharmacology

21 June 2012

Number of times this article has been viewed

\author{
Youssouf Cissél \\ Isao Inoue ${ }^{2}$ \\ Hiroshi Kido' \\ 'Division of Enzyme Chemistry, \\ ${ }^{2}$ Division of Molecular Neurobiology, \\ Institute for Enzyme Research, \\ University of Tokushima, Tokushima, \\ Japan
}

Background: Oseltamivir phosphate (OP, Tamiflu $\left.{ }^{\circledR}\right)$ is a widely used drug in the treatment of influenza with fever. However, case reports have associated OP intake with sudden abnormal behaviors. In rats infected by the influenza A virus (IAV), the electroencephalogram (EEG) displayed abnormal high-voltage amplitudes with spikes and theta oscillations at a core temperature of $39.9^{\circ} \mathrm{C}$ to $41^{\circ} \mathrm{C}$. Until now, there has been no information describing the effect of OP on intact brain hippocampal activity of IAV-infected animals during hyperthermia.

Objective: The aim of the present study was to examine the effect of OP on abnormal EEG activities in the hippocampus using the rat model of influenza-associated encephalopathy.

Methods: Male Wistar rats aged 3 to 4 weeks were used for the study. Influenza A/WSN/33 strain $\left(1 \times 10^{5}\right.$ plaque forming unit in PBS, $\left.60 \mu \mathrm{L}\right)$ was applied intranasally to the rats. To characterize OP effects on the IAV-infected rats, EEG activity was studied more particularly in isofluraneanesthetized IAV-infected rats during hyperthermia.

Results: We found that the hippocampal EEG of the OP-administered (10 mg/kg) IAV-infected rats showed significant reduction of the high-voltage amplitudes and spikes, but the theta oscillations, which had been observed only at $>40^{\circ} \mathrm{C}$ in $\mathrm{OP}$ non-administered rats, appeared at $38^{\circ} \mathrm{C}$ core temperature. Atropine $(30 \mathrm{mg} / \mathrm{kg}$ ) blocked the theta oscillations.

Conclusion: Our data suggest that OP efficiently reduces the abnormal EEG activities after IAV infection during hyperthermia. However, OP administration may stimulate ACh release in rats at normal core temperature.

Keywords: influenza A virus, oseltamivir, electroencephalogram, slow oscillations, theta oscillations, hippocampus

\section{Introduction}

Influenza A virus (IAV) is a common infectious pathogen in humans, which occasionally causes influenza-associated encephalopathy (IAE). IAE is characterized by severe neurological complications, such as convulsive seizures, loss of consciousness, and abnormal behaviors. ${ }^{1,2}$

Oseltamivir phosphate (OP) is a selective neuraminidase inhibitor that prevents influenza virus replication. It is prescribed for seasonal influenza and was the recommended drug for treating the anticipated pandemic of swine influenza (H1N1) in 2009. ${ }^{3-5}$ OP works effectively in humans when used within 48 hours following the first appearance of symptoms (fever). ${ }^{6}$ However, case reports have associated OP intake with sudden abnormal behaviors. ${ }^{7-9}$

Recent studies have shown that OP and its metabolite, OP carboxylate (OC), cross the blood-brain barrier. ${ }^{10,11}$ They have been shown to induce neuronal excitability 
and synchrony in hippocampal slices and slice cultures., ${ }^{72}$ Moreover, OP increases dopamine levels in the medial prefrontal cortex of rats. ${ }^{13}$ Most animal model studies of the action of OP on the brain have been conducted in normal rather than IAV-infected rats or mice, and the animals functioned at the normal core temperature. Until now, there has been no information describing the effect of OP on intact brain hippocampal activity of IAV-infected animals during hyperthermia.

Despite the efficacy of OP in clinics, the putative side effects associated with OP have made the use of this drug controversial. In clinics, IAE patients' electroencephalograms (EEG) are characterized by abnormal high-voltage EEG activity with spikes and theta oscillations during high fever. ${ }^{14-16}$ These clinical abnormal EEG activities (high-voltage amplitudes, increased EEG spikes, and theta oscillations) were reproduced in the rat model of IAE under hyperthermia $\left(39.9^{\circ} \mathrm{C}-41^{\circ} \mathrm{C}\right) .{ }^{17}$

Rats and mice do not normally develop fever after IAV infection. ${ }^{17,18}$ In a previous study, ${ }^{17}$ abnormal EEG activities were not observed in non-anesthetized IAV-infected rats at the normal core temperature $\left(37^{\circ} \mathrm{C}-38^{\circ} \mathrm{C}\right)$. Thus, hyperthermia was a precondition to observe abnormal EEG activities and was induced using a heating system, as previously reported. ${ }^{17}$ In the present study, we examined the effect of OP on abnormal EEG activities in the hippocampus of isoflurane-anesthetized rats during hyperthermia, using the rat model of IAE. ${ }^{17}$

\section{Methods}

\section{Animals, virus infection and electrophysiology}

This study was performed in accordance with the guidelines for animal care and use approved by the animal care committee of The University of Tokushima. Since our goal was to model IAE in children, male Wistar rats aged 3 to 4 weeks were used because rats in this range correspond roughly to the human age range of 2.5 to 3 years. ${ }^{19}$

For viral infection, the rats were anesthetized with ketamine-xylazine $(62.6 \mathrm{mg} / \mathrm{kg}-12.4 \mathrm{mg} / \mathrm{kg})$. Influenza $\mathrm{A} / \mathrm{WSN} / 33$ strain was stocked (frozen) at $-82^{\circ} \mathrm{C}$ and diluted to obtain the dose $\left(1 \times 10^{5}\right.$ plaque forming unit in $\mathrm{PBS}$, $60 \mu \mathrm{L}$ ), which was then applied intranasally to the rats, as previously reported. ${ }^{17}$ The rooms for virus stockage, viral infection, and handling of the infected animals were designed in accordance with the guidelines for animal care and use of The University of Tokushima.

\section{Anesthesia and hyperthermia}

The in vivo electrophysiology experiments were carried out under isoflurane anesthesia. EEG activity in isoflurane anesthetized animals is characterized by slow bursting activity followed by a burst suppression period. ${ }^{17,20}$

The rats were first anesthetized by low-dose of ether, then fitted to a stereotaxic frame (model SN-6N, Narishige, Tokyo). The animals were gas-anaesthetized with 1.5-1.7 minimum alveolar concentration (MAC) of isoflurane mixed with $30 \% \mathrm{O}_{2}$ and $70 \% \mathrm{~N}_{2}$, which allowed spontaneous respiration. During the EEG recordings, a heating pad system (model 21051-00; Fine Science Tools Inc, Foster City, CA) with a feedback control probe was inserted rectally. The body temperature of rats placed on the heating pad gradually increased from $37^{\circ} \mathrm{C}$ to $41^{\circ} \mathrm{C}$ in a period of 20 minutes, as previously reported. ${ }^{17}$

\section{EEG recordings}

EEG activity was recorded using enamel-coated tungsten wire electrodes with an uncoated diameter of $120 \mu \mathrm{m}$ (MT Giken Co, Tokyo, Japan). Craniotomy was performed without damaging the underlying dura using a standard miniature drill equipped with $0.5 \mathrm{~mm}$ diameter drill bit. The electrodes were inserted based on the rat brain in stereotaxic coordinates of Paxinos and Watson. ${ }^{21}$ To record the hippocampal activity, an electrode was lowered into the CA1-CA3 area (left hemisphere) at 2.8 to $3.0 \mathrm{~mm}$ posterior to the bregma, 2.7 to $2.9 \mathrm{~mm}$ lateral from midline, and 2.6 to $3.0 \mathrm{~mm}$ below the dura. Signals were recorded using a dual microprobe system (WP Manufacturing, Inc, Longmont, CO), and a homemade amplifier $(\times 1000)$. The baseline was adjusted to zero-level with a slow voltage clamp system with a time constant of 2.2 seconds. The signal was low-pass filtered at 0.5 to $3 \mathrm{kHz}$, sampled at $1 \mathrm{kHz}$, and recorded using Axopatch software (Axon Instruments, Palo Alto, CA). To verify the electrode position, the electrode tip was coated with a lipophilic tracer dissolved in dimethylsulfoxide at a concentration of $1 \mathrm{mg} / \mathrm{mL}$ to $2.5 \mathrm{mg} / \mathrm{mL}$ before insertion into the brain. After removal of the electrodes, the rats were anesthetized with ketamine-xylazine $(62.6 \mathrm{mg} / \mathrm{kg}-12.4 \mathrm{mg} / \mathrm{kg})$ and were transcardially perfused with saline, followed by fixation ( $4 \%$ paraformaldehyde). The brain was removed and immediately put in sucrose and kept in a $4^{\circ} \mathrm{C}$ room. Sections $800 \mu \mathrm{m}$ thick were then prepared. Red traces of the dye left by the electrodes were observed under the microscope and photographed.

The electrophysiology EEG data analysis was performed using IGOR Pro 4 (WaveMetrics, Inc, Lake Oswego, OR) 
and the fast Fourier transformation (FFT) of EEG activity was computed for 30-second periods.

Slow and distinctive EEG waves were monitored continuously during the experiments to ensure that the rat was well anesthetized and without pain. At the end of experiments, the rats were given a lethal dose of pentobarbital $(50 \mathrm{mg} / \mathrm{kg})$.

\section{Characterization of abnormal EEG activities}

In order to examine the efficacy of OP on abnormal EEG activities during hyperthermia, we focused particularly on the following observed after IAV infection in the rats during hyperthermia: ${ }^{17}$ abnormal high-voltage EEG activity, enhanced low-amplitude fluctuation (LAF) during burst suppressed periods, increased EEG spikes, and theta oscillations. The EEG activity was recorded in the hippocampus of the IAV-infected rats at 8 to 12 hours, 26 to 30 hours, and 50 to 56 hours, corresponding to the periods during which the peak abnormal EEG activities are observed in the rat model of IAE. ${ }^{17}$

The EEG amplitude was measured by quantifying the number of bursts ( $n=15$ bursts average/point) and the EEG spike was identified as a sharp wave that usually sprouts randomly within the burst and during the burst suppressed periods, as previously described. ${ }^{17}$ Theta oscillations amplitude was determined by measuring theta wave amplitude (from the positive to the peak, $n=15$ waves average/points). The details of the EEG components and parameters are shown in Figure 1A.

\section{Drug and antagonist treatment}

All the rats were infected with IAV. OP was not administered (control) to some of the rats, but was administered to the remaining rats. Tamiflu ${ }^{\circledR}$ capsules $(75 \mathrm{mg}$ ) were purchased commercially from Chugai Pharmaceutical Co. (Tokyo, Japan), and the OP in the contents of the capsules was dissolved in water. OP mixture with stabilizing additives from the capsules or recrystallized OP was orally administered to the rats and they were monitored (for 1 hour postadministration) in a cage prior to the EEG recording. OP was administered to the rats in a single dose or in two doses per day (Figure 2A).

Atropine (30 mg/kg; Nacarai, Kyoto, Japan), an mAChR agonist, was dissolved in saline $(0.9 \% \mathrm{NaCl})$ and was given via intraperitoneal (ip) injection.

\section{Statistical analyses}

Numerical values were expressed as mean \pm SD. $P$ value was obtained by Student's paired $t$-test (using SigmaPlot 10; Systat Software Inc, San Jose, CA), and $P<0.05$ was considered statistically significant.

\section{Results}

In single dose cases, OP was administered 3 hours after infection $(n=5)$ and EEG was recorded at 8 hours to 12 hours after infection. In the control rats (IAV-infected rats not given OP), the EEG showed slow oscillations at $38^{\circ} \mathrm{C}$ core temperature; the corresponding slow component $(<1 \mathrm{~Hz})$ is depicted in the FFT plot (Figure $1 \mathrm{~B}$, top left). At $40^{\circ} \mathrm{C}$ core temperature, the EEG showed high-voltage slow EEG activity (or theta-like oscillations in two of the five rats) with increased spikes (arrowheads in the EEG traces) and enhanced amplitudes of the LAF, as shown in the expanded trace (segment 1) (Figure 1B, left bottom). In this study and a previous study, ${ }^{17}$ theta-like oscillations in the control rats were observed only during hyperthermia $\left(39.9^{\circ} \mathrm{C}-41^{\circ} \mathrm{C}\right)$. In contrast, in the OP-administered rats, the EEG displayed theta-like oscillations at the normal core temperature $\left(38^{\circ} \mathrm{C}\right)$ in two out of the five rats (Figure 1B, top right). The FFT plot shows the corresponding theta frequency distributions (Figure 1B, bottom right). At $40^{\circ} \mathrm{C}$, the EEG amplitude was reduced and the rhythmic activity of the LAF was disrupted while the amplitude of the LAF was reduced, as shown in the expanded trace (segment 2 ) shown in Figure 1B. There was a significant difference $(\mathrm{n}=3, P<0.001)$ between the amplitude of LAF values of the OP-administered rats $(0.14 \pm 0.05 \mathrm{mV})$ and that of the control rats $(0.39 \pm 0.06 \mathrm{mV})$. The quantified abnormal EEG amplitudes (left) and EEG spikes (right) were reduced in the OP-administered rats compared with the control rats (Figure 1B, bottom).

The EEG was then recorded at 26 to 30 hours after infection. Abnormal EEG amplitudes in OP-administered rats were reduced $(n=5$, supplementary Figures 1$)$, but the reduction was not significant. However, the amplitude of LAF values $(0.26 \pm 0.04)$ was significantly $(\mathrm{n}=3, P<0.05)$ reduced in the OP-administered rats compared to the control rats $(0.14 \pm 0.04)$, and the EEG spikes were significantly $(P<0.001)$ reduced in OP-administered rats. This suggests that the efficiency of the single-dose OP administration may be weakened after 26 hours of infection.

When two doses of OP were administered per day, abnormal EEG amplitudes $(P<0.05)$ and EEG spikes $(P<0.001)$ recorded at 26 to 30 hours after infection were more significantly reduced compared with the control rats $(\mathrm{n}=3$; Figure $2 \mathrm{~B}$, top). Those at 50 to 60 hours after infection were also reduced in comparison with the control rats $(n=3$, Figure $2 \mathrm{~B}$, bottom). This finding suggests that two doses of $\mathrm{OP}$ administered per day were more efficient than a single dose in reducing abnormal EEG activities in IAV-infected rats. 
A

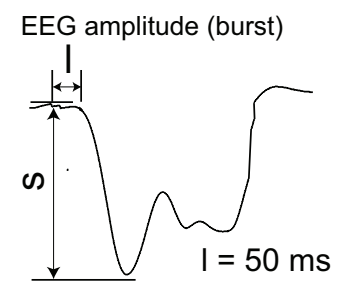

Spike

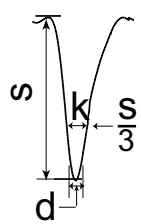

The LAF amplitude (h)

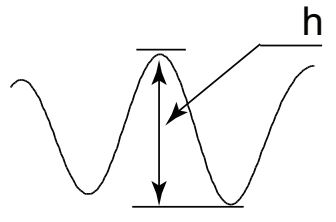

B
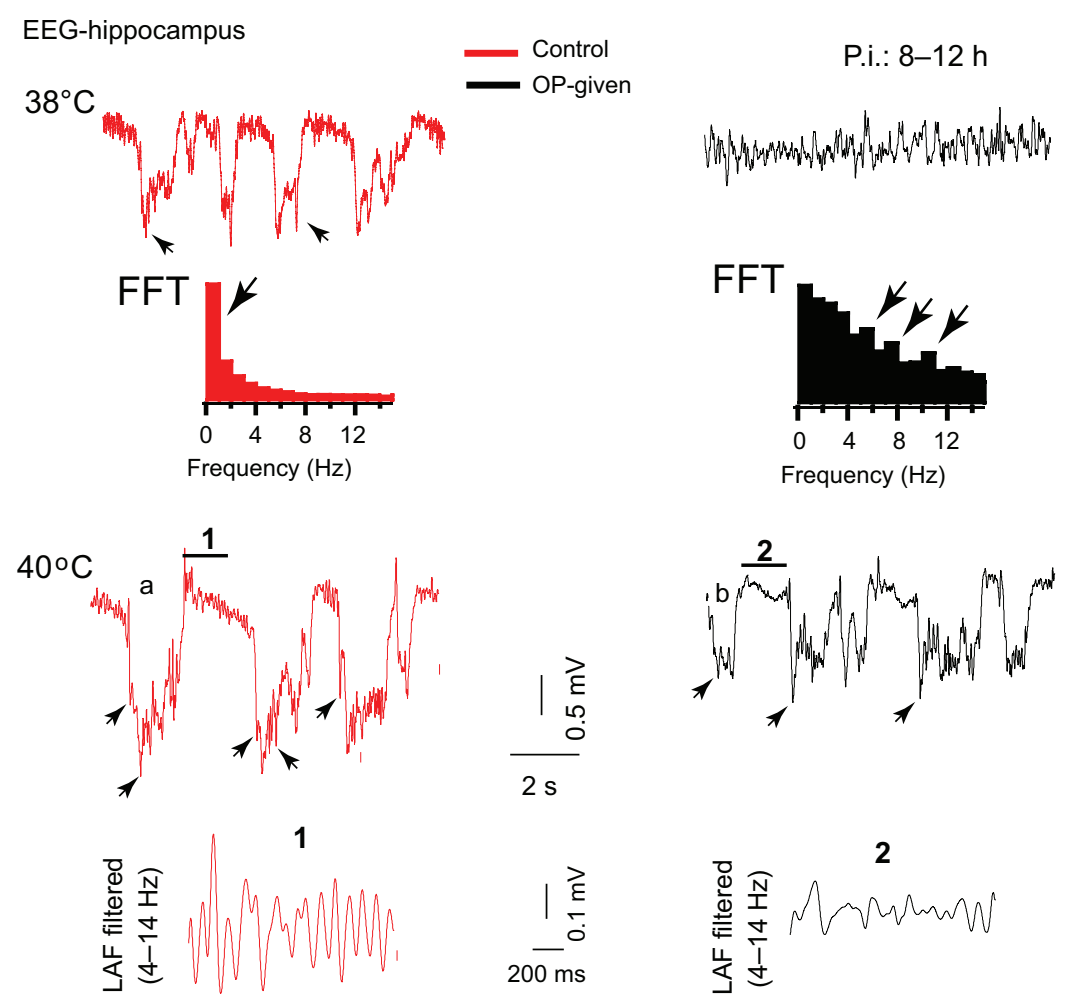

|

$200 \mathrm{~ms}$
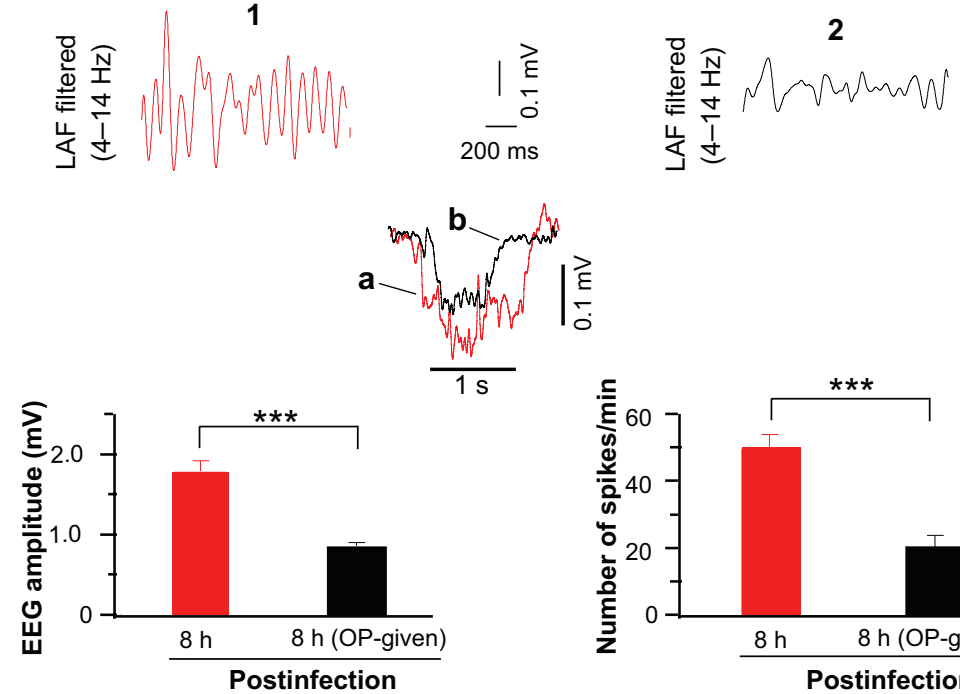

b<smiles>C1CCCCC1</smiles>

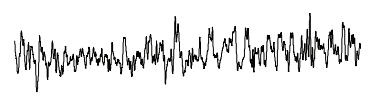


A Two doses administration
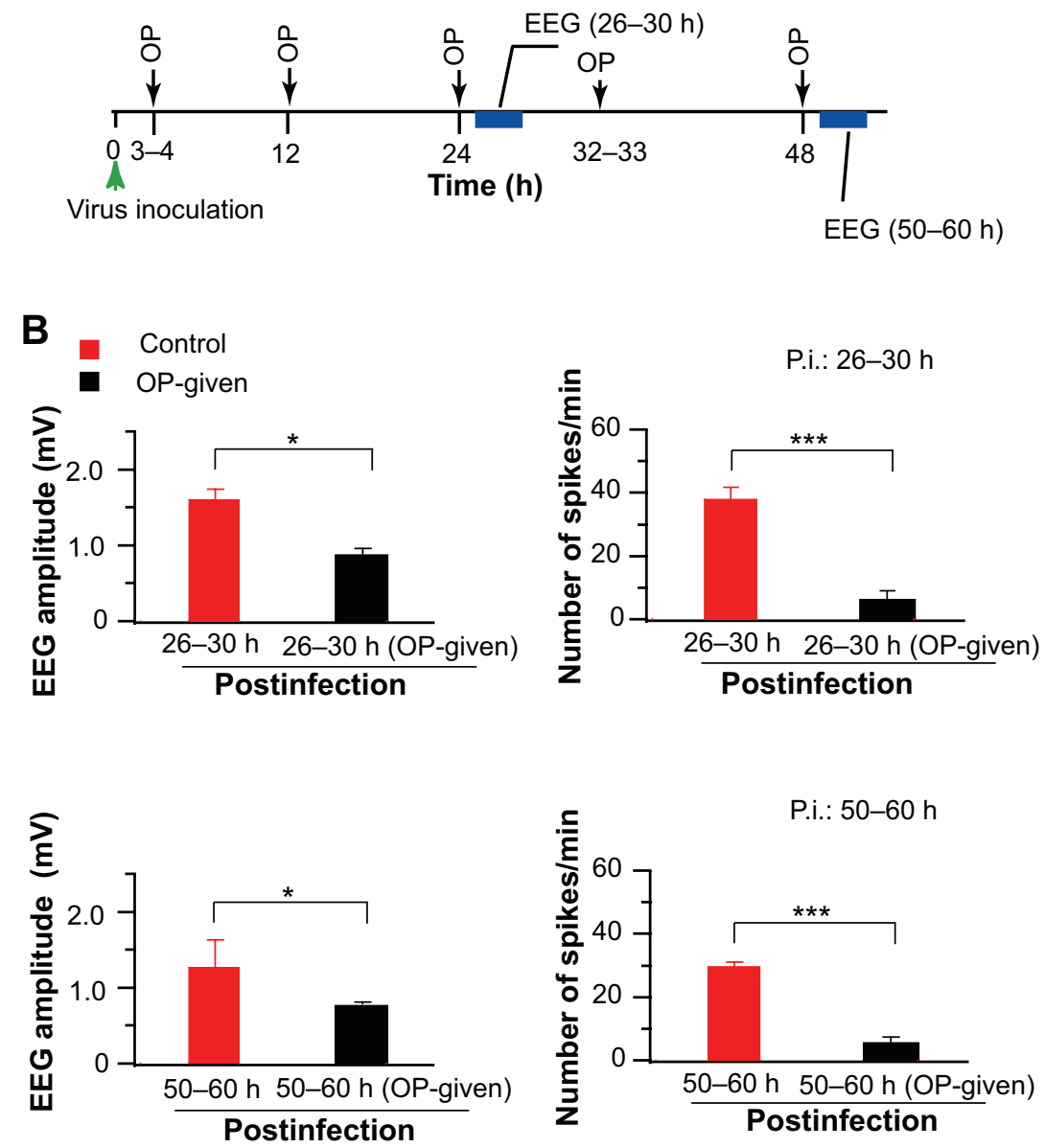

Figure 2 OP significantly reduced abnormal EEG activities. OP applied two doses per day. (A) OP oral administration and EEG recording time course is schematized. The green arrowhead indicates the virus inoculation time, dark arrows show the OP administration time, and the blue rectangle indicates the EEG recording time. (B) Top: EEG activity recorded at 26 to 30 hours after infection.

Notes: The quantification plot shows reduced EEG amplitudes (left) and EEG spikes (right) in the OP-given rats. Bottom: Similar findings and arranged as in top for EEG activity recorded at 50 to 60 hours after infection. OP-given means OP-administered. Data are mean value \pm standard deviation of the mean. $* P<0.05 ; * * * P<0.00 \mathrm{I} ; \mathrm{n}=3$.

Abbreviations: EEG, electroencephalogram; OP, oseltamivir phosphate.

Two types of theta are known: atropine sensitive (a muscarinic receptor blocker) and atropine resistant. ${ }^{22,23}$ We studied the effect of atropine on the OP-induced theta oscillations in IAV-infected rats. We first confirmed that EEG displayed theta oscillations at $38^{\circ} \mathrm{C}$. Then, maintaining this temperature, a saline ip solution was injected, and 30 minutes after the saline injection, atropine $(30 \mathrm{mg} / \mathrm{kg}$, ip) was administered. As can be seen in Figure 3, atropine blocked the theta oscillation in all the rats examined $(n=3)$, revealing that the OP-induced theta oscillation is atropine sensitive.

\section{Discussion}

The present work is the first study to report the effect of OP on rats infected with IAV under hyperthermia in vivo.
We found that one or two orally administered doses of OP were efficient when given soon $(\sim 3$ hours $)$ after IAV infection. OP efficiently reduced the abnormal EEG activities (ie, enhanced amplitude of the LAF, high-voltage EEG amplitudes, increased EEG spike) in the IAV-infected rats. However, we also observed theta oscillations at $38^{\circ} \mathrm{C}$ core temperature in the OP-administered rats.

Although the mechanisms by which OP reduces abnormal EEG activity are not clear, we presume at least that the $\mathrm{N}$-methyl-D-aspartate (NMDA) receptor and the blockage of the virus spread from the host cells could play important roles in reducing the high-voltage amplitudes and EEG spikes, as explained below.

Izumi $^{7}$ reported that $\mathrm{OP}$ induces neuronal excitability via NMDA receptor activation. Our recent study also found that in uninfected rats the activation of NMDA 


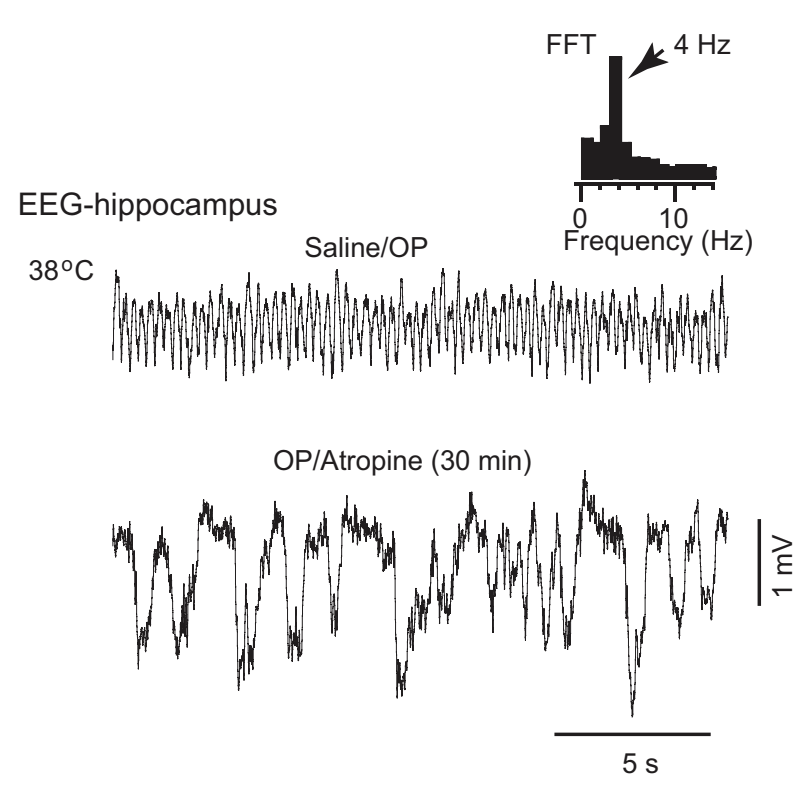

Figure 3 Atropine blocked theta oscillations. The EEG activity was recorded in the hippocampus at $38^{\circ} \mathrm{C}$ rat body temperature. OP-induced theta oscillations were suppressed by intraperitoneal injection of atropine.

Notes: The FFT plot indicating the theta frequency peak at $4 \mathrm{~Hz}$ (top). Atropine blocked theta oscillation in all administered $(n=3)$ rats. FFT of EEG activity was computed for periods of 30 seconds.

Abbreviations: EEG, electroencephalogram; OP, oseltamivir phosphate; FFT, fast Fourier transformation.

receptors peaked at 2 hours after OP administration and declined at 4 hours after OP administration (data not shown). This suggests the inactivation of the NMDA receptors at 4 hours after OP administration, and this inactivation may prevent calcium influx into the neurons. It is also known that OP is a sialic acid analogue, which inhibits the influenza neuraminidase enzyme and prevents the release and spread of the virus from infected host cells during budding. ${ }^{4,6}$ OP treatment (1 hour after infection at daily base) significantly reduces the infection rate in multiple cell types and reduces the progression of the virus in mice. ${ }^{24}$ These previous findings suggest that the early administration of OP after IAV infection within a period before the virus proliferates, in addition to the inactivation of the NMDA receptors at a later time ( $>4$ hours) after OP administration, could be possible factors in explaining how OP works to reduce the abnormal EEG activities.

The observation of theta oscillations at $38^{\circ} \mathrm{C}$ in OPadministered rats is an important finding for confirming the possible action of OP on muscarinic acetylcholine (mACh) receptors. We have observed a similar finding in normal uninfected rats; the $\mathrm{OP}$ administered to these rats induced theta oscillations $(2 \mathrm{~Hz}$ to $6 \mathrm{~Hz})$ at $38^{\circ} \mathrm{C}$ core temperature (data not shown). Theta oscillations were atropine sensitive and prevented fast hippocampal activity, which resulted in a slow EEG activity (Figure 3). This result is in full agreement with a previously reported in vitro study in which OP was found to activate $\mathrm{mACh}$ receptors. ${ }^{12}$ The activation of $\mathrm{mACh}$ receptors may suggest the stimulation of acetylcholine containing cholinergic neurons, which have been known to play important roles in cortical activation and in regulating states of consciousness. ${ }^{25,26} \mathrm{ACh}$ is a major excitatory neurotransmitter in the central nervous system. It plays key roles during synaptic transmission, and constitutes a system with other neurotransmitter/modulators to regulate brain states of vigilance. ${ }^{25}$

Because the EEG recording from the hippocampus in nonanesthetized rats was not possible, the effect of anesthesia could be an issue to consider during the interpretation of the data. Isoflurane has a neuroprotective effect on the brain. It reduces excitatory synaptic transmission in the hippocampus, ${ }^{27-29}$ and it enhances inhibitory synaptic potentials at concentrations above $0.5 \mathrm{MAC}(1 \%){ }^{29}$ Isoflurane alters the $\mathrm{ACh}$ release in a dose-related manner, and that at 1.5 MAC, it significantly decreases ACh release in the cortex and striatum of rats. ${ }^{30,31}$ With such concentrations, EEG activity is generally characterized by slow (bursting) activity followed by burst-suppressed periods. ${ }^{17,20}$ In the present and previous studies, ${ }^{17}$ 1.5-1.7 MAC isoflurane was used. Thus, it is unlikely that the theta oscillations were isoflurane dependent.

The physiological significance of high-voltage slow and theta oscillations in influenza patients in the clinic is not well documented. However, in the rat model of IAE, we speculated that the alternation between these two oscillations may lead to brain instability, and that this may explain the abnormal behaviors observed in some patients. ${ }^{17}$ In the present study, the OP-induced theta oscillations were similar to those observed during hyperthermia in the IAV-infected rats not given OP. Thus, both theta oscillations suggest the stimulation of ACh release. Excessive release of ACh may affect synaptic transmission and oscillation patterns in the brain, which may lead to abnormal behavior in influenza patients. Under such conditions, atropine may play a therapeutic role in stabilizing the brain states from fast $(2-6 \mathrm{~Hz})$ to slow $(<1 \mathrm{~Hz})$ oscillations.

In the present study, because hyperthermia was a precondition for observing EEG abnormalities in the control rats, the effect of OP on core temperature before and during EEG recording was not investigated. However, recent studies showed that OP induces hypothermia in mice ${ }^{32}$ and OP administered in ethanol-injected rats significantly augmented 
the hypothermia effect. ${ }^{7}$ Hypothermia has also been reported after $\mathrm{OP}$ ingestion in clinics. ${ }^{33}$

\section{Conclusion}

Our data show that OP, when administered in the early phase of an infection, can efficiently reduce abnormal EEG activities in IAV-infected rats. However, OP administration may stimulate $\mathrm{ACh}$ release in rats at the normal core temperature.

Future studies should investigate the biomolecular basis of NMDA and mAChRs expression levels and measure ACh release in OP-administered rats, especially in the cortex and the hippocampus. Such an investigation could improve our understanding of protecting the brain during the early phase of IAV infection against neuronal excitability and neurological complications, and thus prevent abnormal behaviors (which can lead to fatal accidents) in influenza patients with high fever.

\section{Acknowledgments}

This research was supported by Grants-in-Aid (20611013) and the Special Coordination Funds for Promoting Science and Technology of the Ministry of Education, Culture, Science and Technology of Japan.

\section{Disclosure}

The authors report no conflicts of interest, direct or indirect, in this work.

\section{References}

1. Kasai T, Togashi T, Morishima T. Encephalopathy associated with influenza epidemics. Lancet. 2000;355:1558-1559.

2. Morishita T, Togashi T, Yokota Y, et al. Encephalitis and encephalopathy associated with an influenza epidemic in Japan. Clin Infect Des. 2002;35: 512-517.

3. Gubareva L, Okomo-Adhiambo M, et al. Update: Drug susceptibility of swine-origin influenza A (H1N1) viruses. Center for Disease Control and Prevention (CDC). MMWR Morb Mortal Wkly Rep. 2009;58: 433-435.

4. Moscona A. Neuraminidase inhibitors for influenza. $N$ Engl $J$ Med. 2005;353:1363-1373.

5. Sawabuchi T, Suzuki S, Iwase C, et al. Boost of mucosal secretory immunologlobulin: a response by clarithromycin in paediatric influenza. Respirology. 2009;14:1173-1179.

6. McNicholl IR, McNicholl JJ. Neuraminidase inhibitors: zanamivir and oseltamivir. Ann Pharmacother. 2001;35:57-70.

7. Izumi Y, Tokuda K, O’Dell KA, Zorumski CF, Narahashi T. Neuroexcitatory action of tamiflu and its carboxylate metabolite. Neurosci Lett. 2007;426:54-58.

8. Maxwell SR. Tamiflu and neuropsychiatric disturbance in adolescents. BMJ. 2007;334:1232-1233.

9. Tanabe T, Hara K, Nakajima M, et al. Oseltamivir treatment for children showing abnormal behavior during influenza virus infection. Brain Dev. 2010;32:440-444.

10. Morimoto K, Nakakariya M, Shirasaka Y, et al. Oseltamivir (Tamiflu ${ }^{\mathrm{TM}}$ ) efflux transport at the blood-brain barrier via P-glycoprotein. Drug Metab Dispos. 2008;36:6-9.
11. Ose A, Kusuhara H, Yamatsugu K, et al. P-glycoprotein restricts the penetration of oseltamivir across the blood-brain barrier. Drug Metab Dispos. 2008;36:427-434.

12. Usami A, Sasaki T, Satoh N, et al. Oseltamivir enhances hippocampal network synchronization. J Pharmacol Sci. 2008;106:659-662.

13. Yoshino T, Nisijima K, Shioda K, Yui K, Kato S. Oseltamivir (Tamiflu) increases dopamine levels in the rat medial prefrontal cortex. Neurosci Lett. 2008;438:67-69.

14. Sato S, Kumada S, Koji T, Okaniwa M. Reversible frontal lobe syndrome with influenza virus infection in children. Pediatr Neurol. 2000;22(4):318-321.

15. Okumura A, Nakano T, et al. Delirious behavior in children with influenza: its clinical features and EEG findings. Brain Dev. 2005;27:271-274.

16. Fukumoto Y, Okumura A, Fukumoto K, et al. Serum levels of cytokines and EEG finding in children with influenza associated with mild neurological complications. Brain Dev. 2007;29:425-430.

17. Cissé Y, Wang S, Inoue I, Kido H. Rat model of influenza-associated encephalopathy (IAE): study of electroencephalogram (EEG) in vivo. Neurosci. 2010;165:1127-1137.

18. Toth LA, Rehg JE, Webster RG. Strain differences in sleep and other pathophysiological sequelae of influenza virus infection in naive and immunized mice. J Neuroimmunol. 1995;58:89-99.

19. Ruth EB. Metamorphosis of the public symphysis. I. The white rat (Mus norgicus albinos). Anat Rec. 1935;64:1-7.

20. Kroeger D, Amzica F. Hypersensitivity of anesthesia-induced comatose brain. J Neurosci. 2007;27(39):10597-10607.

21. Paxinos P, Watson C. The Rat Brain in Stereotaxic Coordinates, 5 th ed. Amsterdam; Boston: Elsevier Academic Press; 2005.

22. Goutagny R, Jackson J, Williams S. Self-generated theta oscillations in the hippocampus. Nat Neurosci. 2009;12(12):1491-1493.

23. Kramis R, Vanderwolf $\mathrm{CH}$, Bland BH. Two types of hippocampal rhythmical slow activity in both the rabbit and the rat: Relations to the behaviour and effects of atropine, diethyl ether, urethane, and pentobarbital. Exp Neurol. 1975;49(1 Part 1):58-85.

24. Manicassamy B, Manicassamy S, Belicha-Villanueva A, et al. Analysis of in vivo dynamics of influenza virus infection in mice using a GFP reporter virus. PNAS. 2010;107(25):11519-11524.

25. Jones BE. Neurotransmitter systems regulating sleep-wake states. Biological Psychiatry. In: D'Haenin D, den Boer JA, Willner P, editors. New York: John Wiley and Sons; 2002;2:1215-1228.

26. Celesia GG, Jasper HH. Acetylcholine released from cerebral cortex in relation to state of activation. Neurology. 1996;16:1053-1063.

27. Lloyd-Thomas AR, Cole PV, Prior PF. Quantitative EEG and brainstem auditory evoked potentials: Comparison of isoflurane with halothane using the cerebral function analsysing monitor. Br J Anaesth. 1990;65:306-312.

28. MacIver MB, Roth SH. Inhalation anaesthetics exhibit pathway-specific and differential actions on hippocampal synaptic responses in vitro. $\mathrm{Br}$ J Anaesth. 1988;60:680-691.

29. Miu P, Puil E. Isoflurane-induced impairment of synaptic transmission in hippocampal neurons. Exp Brain Res. 1989;75:354-360.

30. Shichino T, Murakawa M, Adachi T, Miyazaki Y, Mori K. Effects of inhalation anaesthetics on the release of acetylcholine in the rat cerebral cortex in vivo. Br J Anaesth. 1998;80:365-370.

31. Shichino T, Murakawa M, Adachi T, et al. Effects of isoflurane on in vivo release of acetylcholine in the rat cerebral cortex and striatum. Acta Anesthesiol Scand. 1997;41:1335-1340.

32. Ono H, Nagano Y, Matsunami N, et al. Oseltamivir, an antiinfluenza virus drug, produces hypothermia in mice: comparison among oseltamivir, zanamivir and diclofenac. Biol Pharm Bull. 2008;31:638-642.

33. Ministry of Health, Labor and Welfare, Japan (MHLW). Public opening data (documents 5-1-1 and 5-2) at the Meeting for Safety Measure Investigation Council, April 4, 2007. Available from: http://www. mhlw.go.jp/shingi/2007/04/s0404-2.html. Accessed April 4, 2007. Japanese. 


\section{Supplementary figure}

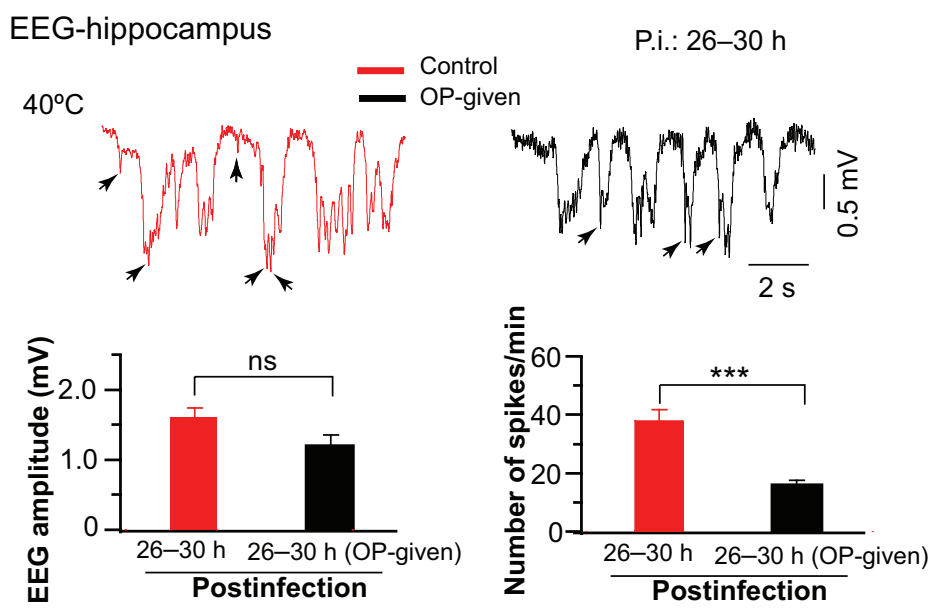

Figure SI OP significantly reduced EEG spikes but not EEG amplitudes.

Notes: The effect of OP was weakened after 26 to 30 hours postinfection. Note a decrease of EEG amplitude in the OP-administered rats compared with the control rats. However, the difference was not significant. In contrast, a significant decrease in the number of spikes was observed in the OP-administered rats.

Abbreviations: OP, oseltamivir phosphate; EEG, electroencephalogram.

\section{Publish your work in this journal}

The Journal of Experimental Pharmacology is an international, peerreviewed, open access journal publishing original research, reports, reviews and commentaries on all areas of laboratory and experimental pharmacology. The manuscript management system is completely online and includes a very quick and fair peer-review system.
Visit http://www.dovepress.com/testimonials.php to read real quotes from published authors. 